\title{
Improved finite element of a transversely cracked straight beam with an additional degree of freedom
}

\author{
Denis Imamovića* (i) \\ Matjaž Skrinar ${ }^{a}$ ๑ \\ a Faculty of Civil Engineering, Transportation Engineering and Architecture, University of Maribor, Smetanova 17, Maribor, Slovenia. \\ E-mail: denis.imamovic@um.si, matjaz.skrinar@um.si \\ *Corresponding author
}

http://dx.doi.org/10.1590/1679-78255337

\begin{abstract}
A new finite element with an additional degree of freedom at the crack location is derived for static bending analysis of a transversely cracked uniform slender beam. In the simplified computational model, which is based on Euler-Bernoulli's theory of small displacements, the crack is represented by a linear rotational spring connecting two elastic parts. The derivation of the transverse displacements, the coefficients of the stiffness matrix as well as the load vector for uniformly distributed load along the whole beam element was based on the utilization of polynomial interpolation functions of the fourth degree and all derived expressions were obtained in the closed form. The novelty of the new model, by comparison to the previously presented simplified finite element models, is that the transverse displacements functions obtained by utilization of the newly presented interpolation functions for the case of uniform continuous transverse load along whole beam element, as well as the functions of the bending moments and transverse forces, are accurate. The values obtained by the simplified model also exhibited good agreement in additional comparison with the results from more demanding and more detailed 2D models.
\end{abstract}

\begin{abstract}
Keywords:
Bending of beams with transverse cracks, Closed-form solutions, Euler-Bernoulli beam, Finite element method, Load vector, Simplified computational model, Static analysis, Stiffness matrix, Transverse displacements.
\end{abstract}

\section{INTRODUCTION}

Any degenerative effect that might occur in structures during their utilization can severely decrease structures' stiffness and potentially lead to their failure. Bearing this in mind, several studies aimed at the detection and identification of damages in engineering structures were conducted. Since the occurrence of damage can alter the structures' response parameters, the structural health monitoring and damage detection methods techniques are commonly based on measured structure's response. However, the efficiency of these approaches depends not only on the measured data's quality but also on the consistencies and versatilities of computational models implemented for mechanical behavior's modelling. Detailed 2D or 3D meshes of finite elements that certainly offer the finest description of a general structure, as well as of the crack and its surrounding location, appear to be uncomfortable and, therefore, simplified models are usually implemented in structural health monitoring techniques.

The model that has been the subject of numerous researches in the past, is the model provided by Okamura et al. (1969). As a crack in a structural member alters the local compliance of rotations, each crack in this model is replaced by a massless rotational linear spring. For the spring of suitable stiffness, the linear moment-rotation constitutive law is adopted. Each spring connects those neighboring non-cracked parts of the beam that are modelled as elastic elements. Several definitions for rotational spring stiffness can be found in the references while the earliest definition of rotational 
spring stiffness for a rectangular cross-section was introduced by Okamura et al. (1969). In addition, some other researchers (Dimarogonas and Papadopulus (1983); Rajab and Al-Sabeeh, 1991; Ostachowicz and Krawczuk (1990); Sundermeyer and Weaver (1995); Hasan (1995); Skrinar and Pliberšek (2004)) have presented their definitions.

Although the simplified model has been primarily developed for direct analysis it is - due to its simplicity - frequently used also in the field of inverse identification of cracks or other local stiffness reductions (Mazanoglu (2015); Caddemi and Caliò (2014)).

Many of the earliest studies reported in the references regarding the behavior of cracked beams were oriented to the exact solution of the simplified model's governing differential equation. However, this approach has severe limitations (except for rather basic structures with simple boundary conditions) as analytical exact governing differential equations' solutions are generally either difficult or even impossible to obtain. Therefore, after the pioneering works by Tharp (1987) and Gounaris and Dimarogonas (1988) for a beam element with a single transverse crack the research interest has oriented towards a finite element solution for bending problems.

Recent researches have also confirmed that the simplified model can be successfully implemented for cracked beams on elastic foundations (Skrinar and Imamović (2018); Alijani et al. (2018)).

Some latest investigations have expanded the research interest from beams with a single crack to multi cracked beams. Several papers were devoted to Euler-Bernoulli beam's finite element having an arbitrary number of transverse cracks differing in the mathematical approaches applied to obtain closed-form solutions for stiffness matrix (Biondi and Caddemi (2007); Skrinar (2009 and 2013); Palmeri and Cicirello (2011); Skrinar and Pliberšek (2012)).

Although these approaches directly lead to exact displacements at element's nodes, this changes when the displacements between the nodes are being evaluated. Standard interpolation functions are complete polynomials of the third degree and, therefore, in situations where the transverse-distributed load is applied, they cannot produce accurate results due to the too low polynomials' degree. Consequently, the governing differential equations' solutions are commonly used alternative to obtain the accurate transverse displacements.

In this paper, the degree of polynomials, implemented as interpolation functions, was increased by introducing a supplementary degree of freedom - transverse displacement in an additional node located at the crack position.

This approach results in standard interpolation functions in the form of complete polynomials of the fourth degree which now produce exact transverse displacements for the most frequent type of transverse load - uniform load. All the derived functions, as well as the stiffness matrix and load vector are given as closed-form solutions. The additional discrete displacement information, in conjunction with novel interpolation functions, now offers the direct evaluation of transverse displacements for both sections to the left and right of the crack.

\section{IMPROVED FINITE ELEMENT MODEL FORMULATION OF A CRACKED BEAM}

In the derivation of the new finite element for the computation of transverse displacements of a cracked homogeneous Euler-Bernoulli beam element, the crack is modelled as a rotational linear-spring connecting two elastic non-cracked parts. This mathematical model was given by Okamura et al. (1969), where the crack is defined by the location (i.e. the distance $L_{1}$ from the left-end) and depth $d$, which determines the stiffness of the rotational spring, indicated as $K_{r}$. This stiffness $K_{r}$ models the remaining flexural rigidity of the cracked cross-section. From all available definitions the one given by Okamura et al. (1969) was applied in this study. It has the following form:

$$
\mathrm{K}_{\mathrm{r}}=\frac{\mathrm{EI}}{\mathrm{h} \cdot 6 \cdot\left(1-\mathrm{v}^{2}\right) \cdot \mathrm{F}\left(\delta^{\prime}\right)}
$$

and it depends on the height of the non-cracked cross-section $h$, the relative depth of the crack $\delta^{\prime}=d / h$, the product of Young's modulus $E$ with a moment of inertia I of the non-cracked cross-section (i.e. flexural rigidity $E$ ) and flexibility compliance function of crack $\mathrm{F}\left(\delta^{\prime}\right)$ derived for the rectangular beam as:

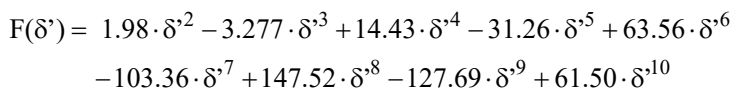

Unlike the other definitions, the genuine definition of the Okamura et al. includes also Poisson's coefficient $v$. 


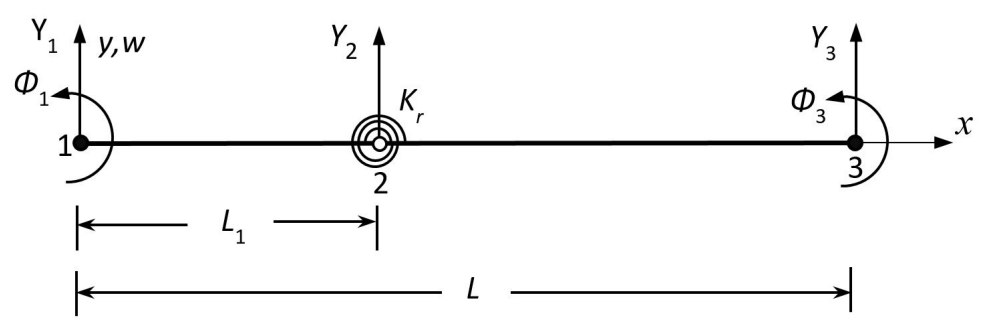

Figure 1: The beam finite-element with a crack represented by a linear rotational spring.

Presented improved finite element (CB3NFE), Figure 1, has three nodes (node 2, positioned at the location of the crack, as a supplement to both standard nodes 1 and 3, located at the beginning and end of the element,) and a total of five degrees of freedom: transverse displacement $Y_{1}$ and rotation $\Phi_{1}$ in the initial (left) node, transverse displacement $Y_{2}$ at the crack (at the distance $L_{1}$ from the left node), as well as transverse displacement $Y_{3}$ and rotation $\Phi_{3}$ at the right end of the element. Upward translations and anticlockwise rotations are taken as positive.

\section{DERIVATIONS}

\subsection{Derivation of the cracked beam's interpolation functions}

Since the crack separates the two elastic parts, the transverse displacements along the length of the beam can no longer be written by a single function. Thus different displacement functions for the left part $\left(w_{1}(x)\right)$ and the right part $\left(w_{2}(x)\right)$ of the crack are required. The functions $w_{1}(x)$ and $w_{2}(x)$ of transverse displacements are complete polynomials of the fourth degree with a total of ten unknown constants $\left(A_{1}, A_{2}, A_{3}, A_{4}, A_{5}, B_{1}, B_{2}, B_{3}, B_{4}\right.$, and $\left.B_{5}\right)$ :

$$
\begin{array}{ll}
\mathrm{w}_{1}(\mathrm{x})=\mathrm{A}_{1}+\mathrm{A}_{2} \cdot \mathrm{x}+\mathrm{A}_{3} \cdot \mathrm{x}^{2}+\mathrm{A}_{4} \cdot \mathrm{x}^{3}+\mathrm{A}_{5} \cdot \mathrm{x}^{4} & \left(0 \leq \mathrm{x} \leq \mathrm{L}_{1}\right) \\
\mathrm{w}_{2}(\mathrm{x})=\mathrm{B}_{1}+\mathrm{B}_{2} \cdot \mathrm{x}+\mathrm{B}_{3} \cdot \mathrm{x}^{2}+\mathrm{B}_{4} \cdot \mathrm{x}^{3}+\mathrm{B}_{5} \cdot \mathrm{x}^{4} \quad\left(\mathrm{~L}_{1} \leq \mathrm{x} \leq \mathrm{L}\right)
\end{array}
$$

To implement functions from Equations (3) and (4) as interpolation (shape) functions for the finite element, the unknown constants have to be determined. Three of them $\left(B_{3}, B_{4}\right.$, and $\left.B_{5}\right)$ can be determined from the condition that the bending moments and shear forces functions for both elastic sections are identical over the whole length of the element:

$\mathrm{w}_{1}{ }^{\prime \prime}(\mathrm{x})=\mathrm{w}_{2}{ }^{\prime \prime}(\mathrm{x}) \quad(0 \leq \mathrm{x} \leq \mathrm{L})$

which initially yields:

$\mathrm{B}_{3}=\mathrm{A}_{3}$

$\mathrm{B}_{4}=\mathrm{A}_{4} \quad(6)$

$\mathrm{B}_{5}=\mathrm{A}_{5}$

Next five unknown constants $\left(A_{1}, A_{2}, A_{3}, A_{4}\right.$, and $\left.A_{5}\right)$ are determined from the kinematic conditions:

$\mathrm{w}_{1}(0)=\mathrm{Y}_{1}$

$\mathrm{w}_{1}^{\prime}(0)=\Phi_{1}$

$\mathrm{w}_{1}\left(\mathrm{~L}_{1}\right)=\mathrm{Y}_{2}$

$\mathrm{w}_{2}(\mathrm{~L})=\mathrm{Y}_{3}$

$\mathrm{w}_{2}{ }^{\prime}(\mathrm{L})=\Phi_{3}$ 
The remaining two constants $\left(B_{1}\right.$ and $\left.B_{2}\right)$ are obtained from continuity conditions at the crack location $\left(x=L_{1}\right)$, where the influence of the crack is presented as the discontinuity of the rotations. These conditions are the equilibrium of the displacements and the difference in the rotations at the crack location, Equations (12) and (13):

$$
\begin{aligned}
& \mathrm{w}_{1}\left(\mathrm{~L}_{1}\right)=\mathrm{w}_{2}\left(\mathrm{~L}_{1}\right) \\
& \mathrm{w}_{1}{ }^{\prime}\left(\mathrm{L}_{1}\right)+\psi \cdot \mathrm{w}_{1}{ }^{\prime}\left(\mathrm{L}_{1}\right)=\mathrm{w}_{2}{ }^{\prime}\left(\mathrm{L}_{1}\right)
\end{aligned}
$$

where $\psi$ is the ratio between the flexural rigidity of the non-cracked cross section $E I$ and the stiffness of the rotational springs $K_{r}$, i.e.:

$$
\psi=\frac{\mathrm{EI}}{\mathrm{K}_{\mathrm{r}}}
$$

Afterwards, when all the coefficients of both sections of the transverse displacements functions are defined, each function can be rewritten as a product of the corresponding vector of interpolation functions $\mathbf{N}_{i}(i=1,2)$ with the vector of unknown nodal displacements q:

$$
\begin{array}{ll}
\mathrm{w}_{1}(\mathrm{x})=\left\{\mathrm{N}_{11}, \mathrm{~N}_{21}, \mathrm{~N}_{31}, \mathrm{~N}_{41}, \mathrm{~N}_{51}\right\} \cdot\left\{\mathrm{Y}_{1}, \Phi_{1}, \mathrm{Y}_{2}, \mathrm{Y}_{3}, \Phi_{3}\right\}^{\mathrm{T}}=\mathrm{N}_{1} \cdot \mathbf{q} & \left(0 \leq \mathrm{x} \leq \mathrm{L}_{1}\right) \\
\mathrm{w}_{2}(\mathrm{x})=\left\{\mathrm{N}_{12}, \mathrm{~N}_{22}, \mathrm{~N}_{32}, \mathrm{~N}_{42}, \mathrm{~N}_{52}\right\} \cdot\left\{\mathrm{Y}_{1}, \Phi_{1}, \mathrm{Y}_{2}, \mathrm{Y}_{3}, \Phi_{3}\right\}^{\mathrm{T}}=\mathrm{N}_{2} \cdot \mathbf{q} \quad\left(\mathrm{L}_{1} \leq \mathrm{x} \leq \mathrm{L}\right)
\end{array}
$$

where the interpolation functions $N_{i 1}$ and $N_{i 2}(i=1,2, \ldots 5)$ are given as (definitions of coefficients $A_{i j}$ and $B_{i j}$ are given in the Appendix A):

$$
\begin{array}{ll}
\mathrm{N}_{\mathrm{i} 1}(\mathrm{x})=\sum_{\mathrm{j}=1}^{5} \mathrm{~A}_{\mathrm{ij}} \mathrm{x}^{\mathrm{j}-1} \quad\left(0 \leq \mathrm{x} \leq \mathrm{L}_{1}\right) \\
\mathrm{N}_{\mathrm{i} 2}(\mathrm{x})=\sum_{\mathrm{j}=1}^{5} \mathrm{~B}_{\mathrm{ij}} \mathrm{x}^{\mathrm{j}-1} \quad\left(\mathrm{~L}_{1} \leq \mathrm{x} \leq \mathrm{L}\right)
\end{array}
$$

\subsection{Derivation of the stiffness matrix coefficients of the cracked beam}

The coefficients of the stiffness matrix $(i, j=1,2, \ldots, 5)$ for the three nodded finite element of the cracked beam (CB3NFE), which were obtained on the basis of deformation energy, are by means of interpolation functions of the fourth degree determined as:

$$
\begin{aligned}
& \mathrm{k}_{\mathrm{ij}}=\mathrm{EI} \int_{\mathrm{x}=0}^{\mathrm{L}_{1}} \mathrm{~N}_{\mathrm{i} 1}{ }^{\prime \prime}(\mathrm{x}) \mathrm{N}_{\mathrm{j} 1}{ }^{\prime \prime}(\mathrm{x}) \mathrm{dx}+\mathrm{EI} \int_{\mathrm{x}=\mathrm{L}_{1}}^{\mathrm{L}} \mathrm{N}_{\mathrm{i} 2}{ }^{\prime \prime}(\mathrm{x}) \mathrm{N}_{\mathrm{j} 2}{ }^{\prime \prime}(\mathrm{x}) \mathrm{dx} \\
& +\mathrm{K}_{\mathrm{r}}\left(\mathrm{N}_{\mathrm{i} 1}{ }^{\prime}\left(\mathrm{L}_{1}\right)-\mathrm{N}_{\mathrm{i} 2}{ }^{\prime}\left(\mathrm{L}_{1}\right)\right)\left(\mathrm{N}_{\mathrm{j} 1}{ }^{\prime}\left(\mathrm{L}_{1}\right)-\mathrm{N}_{\mathrm{j} 2}{ }^{\prime}\left(\mathrm{L}_{1}\right)\right)
\end{aligned}
$$

When introducing Equation (5) into Equation (19) both integrals are replaced by a single integral over the whole length. After evaluating Equation (19) the coefficients of the stiffness matrix are now given as:

$$
\mathrm{k}_{\mathrm{ij}}=\mathrm{EI} \sum_{\mathrm{m}=3}^{5} \sum_{\mathrm{n}=3}^{5}\left(\left(\prod_{\mathrm{k}=1}^{2}(\mathrm{~m}-\mathrm{k})(\mathrm{n}-\mathrm{k})\right) \frac{\mathrm{L}^{\mathrm{m}+\mathrm{n}-5} \mathrm{~A}_{\mathrm{im}} \mathrm{A}_{\mathrm{jn}}}{\mathrm{m}+\mathrm{n}-5}\right)+\mathrm{K}_{\mathrm{r}}\left(\mathrm{A}_{\mathrm{i} 2}-\mathrm{B}_{\mathrm{i} 2}\right)\left(\mathrm{A}_{\mathrm{j} 2}-\mathrm{B}_{\mathrm{j} 2}\right)
$$

\subsection{Derivation of equivalent nodal-force vector for continuous load $p(x)$}

The substitutive nodal load, which replaces the genuinely continuous distributed load $p(x)$ (upward positive) along the finite element, is derived from the condition of work-equivalency. The general substitutive load vector $\mathbf{F}_{p(x)}$ due to distributed transverse load $p(x)$ is thus obtained as a sum of two parts: 


$$
\mathbf{F}_{p(x)}=\int_{x=0}^{L_{1}} p(x) \mathbf{N}_{1} d x+\int_{x=L_{1}}^{L} p(x) \mathbf{N}_{2} d x
$$

or expressed by its coefficients $(i=1,2, \ldots, 5)$ :

$$
F_{p(x), i}=\int_{x=0}^{L_{1}} p(x) N_{i 1}(x) d x+\int_{x=L_{1}}^{L} p(x) N_{i 2}(x) d x
$$

Finally, the coefficients of load vector $(i=1,2 \ldots, 5)$ for a uniformly continuous load $p$ over the whole length $L$ of the element are thus given as:

$F_{p, i}=p \sum_{j=1}^{5} \frac{L_{1}^{j} A_{i j}+\left(L^{j}-L_{1}^{j}\right) B_{i j}}{j}$

\subsection{Evaluation of the nodal forces and bending moments of the external nodes}

The assembling of both, the structure's stiffness matrix and the load vector, allows us to obtain the nodal displacements and rotations for all the finite elements. To evaluate the shear forces and the bending moments at the both ends of the element, the corresponding stiffness matrix $\mathbf{K}$ is multiplied by the element's vector of corresponding nodal displacements and rotations $\mathbf{q}$ and from the product any potential vector of the substitutive nodal loads $\mathbf{F}=\left\{\mathrm{F}_{1}, \mathrm{~F}_{2}, \mathrm{~F}_{3}, \mathrm{~F}_{4}, \mathrm{~F}_{5}\right\}$ of the element is subtracted.

\subsection{Evaluation of the transverse displacements, bending moments and shear forces distribution between nodes}

The transverse displacements' function for each finite element can be written by utilization of Equations (15) and (16) as the product of a vector of interpolation functions and known nodal displacements. The following generalized form is obtained:

$$
\begin{array}{ll}
\mathrm{w}_{1}(\mathrm{x})=\sum_{\mathrm{i}=1}^{5} \mathrm{q}_{\mathrm{i}} \sum_{\mathrm{j}=1}^{5} \mathrm{~A}_{\mathrm{ij}} \mathrm{x}^{\mathrm{j}-1} \quad\left(0 \leq \mathrm{x} \leq \mathrm{L}_{1}\right) \\
\mathrm{w}_{2}(\mathrm{x})=\sum_{\mathrm{i}=1}^{5} \mathrm{q}_{\mathrm{i}} \sum_{\mathrm{j}=1}^{5} \mathrm{~B}_{\mathrm{ij}} \mathrm{x}^{\mathrm{j}-1} \quad\left(\mathrm{~L}_{1} \leq \mathrm{x} \leq \mathrm{L}\right)
\end{array}
$$

where $q_{i}$ represents the $i$-th term (transverse displacement or rotation) of the finite element's displacement vector.

The utilization of Equations (24) and (25) for the evaluation of the transverse displacements' functions for both elastic parts leads to accurate displacement functions for the two most frequent load cases in structural analysis. In the first case, the finite element is without any distributed transverse load whereas in the second case uniformly distributed transverse load is applied across the entire finite element.

Furthermore, the exact functions of the bending moments and shear forces for load cases with and without a uniform transverse load are obtained by means of the second and third derivatives of any of the Equations (24) or (25), which leads to following expressions:

$$
\begin{array}{ll}
\mathrm{M}(\mathrm{x})=\mathrm{EI} \sum_{\mathrm{i}=1}^{5} \mathrm{q}_{i} \sum_{\mathrm{j}=3}^{5}\left(\prod_{\mathrm{m}=1}^{2}(\mathrm{j}-\mathrm{m})\right) \mathrm{A}_{\mathrm{ij}} \mathrm{x}^{\mathrm{j}-3} \quad(0 \leq \mathrm{x} \leq \mathrm{L}) \\
\mathrm{V}(\mathrm{x})=\mathrm{EI} \sum_{\mathrm{i}=1}^{5} \mathrm{q}_{\mathrm{i}} \sum_{\mathrm{j}=4}^{5}\left(\prod_{\mathrm{m}=1}^{3}(\mathrm{j}-\mathrm{m})\right) \mathrm{A}_{\mathrm{ij}} \mathrm{x}^{\mathrm{j}-4} \quad(0 \leq \mathrm{x} \leq \mathrm{L})
\end{array}
$$

\section{NUMERICAL EXAMPLES}

In order to verify the presented finite element, the implementation of derived expressions for the stiffness matrix, the load vector, and the transverse displacements functions from the polynomial interpolation functions of the fourthdegree was demonstrated on two examples. In these examples the transverse displacements along the length of the 
beam, bending moments and shear forces at nodal points, likewise, their functions along the whole length of the beam were examined. Beside the CB3FE model's discretizations three other approaches were implemented (conventional twonode finite element, CB2NFE; solutions of governing differential equations, GDEs; plane finite elements, 2D FE).

Firstly, the new finite element's results were compared to the values obtained by the standard two-nodded cracked beam's finite element (CB2NFE). This element has four degrees of freedom and its interpolation functions are polynomials of the third degree. Its corresponding stiffness matrix and load vector can be found in various forms in different references (for example Skrinar (2013)).

The exact functions of the transverse displacements within the considered structures, which served for additional control of both examples, were obtained from the general solutions of the GDEs of a uniform beam with a continuously distributed transverse load $p$. The differential equation of bending for the cracked beam element is based on standard Bernoulli-Euler theory where different solutions are obtained for the parts to the left and to the right of the crack. These solutions are obtained from two coupled differential equations where standard boundary conditions, as well as continuity conditions as presented by Okamura, are applied. The general form of GDE's solution for each elastic segment $(i=1,2)$ can be written as (Skrinar (2013)):

$$
\mathrm{w}_{\mathrm{GDE}, \mathrm{i}}(\mathrm{x})=\mathrm{C}_{\mathrm{i}}+\mathrm{D}_{\mathrm{i}} \mathrm{x}+\mathrm{E}_{\mathrm{i}} \mathrm{x}^{2}+\mathrm{G}_{\mathrm{i}} \mathrm{x}^{3}+\frac{\mathrm{p}}{24 \mathrm{EI}} \mathrm{x}^{4}
$$

The eight unknown coefficients for both elastic segments were determined (by additional computations) either from the left side boundary conditions of the finite element $\left(\mathrm{w}_{\mathrm{GDE}, 1}(0)=\mathrm{Y}_{1}, \mathrm{w}_{\mathrm{GDE}, 1}{ }^{\prime}(0)=\Phi_{1}, \mathrm{EI}_{\mathrm{GDE}, 2}{ }^{\prime \prime}(0)=\mathrm{F}_{2},-\mathrm{EI}_{\mathrm{GDE}, 1}{ }^{\prime \prime}(0)=\mathrm{F}_{1}\right)$ or the conditions of the continuity between both elastic segments $\left(\mathrm{w}_{\mathrm{GDE}, 1}\left(\mathrm{~L}_{1}\right)=\mathrm{w}_{\mathrm{GDE}, 2}\left(\mathrm{~L}_{1}\right), \mathrm{w}_{\mathrm{GDE}, 1}{ }^{\prime}\left(\mathrm{L}_{1}\right)+\psi \cdot \mathrm{w}_{\mathrm{GDE}, 1}{ }^{\prime}\left(\mathrm{L}_{1}\right)=\mathrm{w}_{\mathrm{GDE}, 2}\left(\mathrm{~L}_{1}\right)\right.$ , $\left.\mathrm{w}_{\mathrm{GDE}, 1} "\left(\mathrm{~L}_{1}\right)=\mathrm{w}_{\mathrm{GDE}, 2} "\left(\mathrm{~L}_{1}\right), \mathrm{w}_{\mathrm{GDE}, 1}{ }^{\prime \prime}\left(\mathrm{L}_{1}\right)=\mathrm{w}_{\mathrm{GDE}, 2}{ }^{\prime \prime}\left(\mathrm{L}_{1}\right)\right)$. The remaining boundary conditions at the right end $\left(\mathrm{w}_{\mathrm{GDE}, 2}(\mathrm{~L})=\mathrm{Y}_{3}\right.$, $\left.\mathrm{w}_{\mathrm{GDE}, 2}{ }^{\prime}(\mathrm{L})=\Phi_{3}, \quad-\mathrm{EIw}_{\mathrm{GDE}, 2}{ }^{\prime \prime}(\mathrm{L})=\mathrm{F}_{5}, \quad \mathrm{EIw}_{\mathrm{GDE}, 2}{ }^{\prime \prime}(\mathrm{L})=\mathrm{F}_{4}\right)$, which were not implemented in the transverse displacement functions' calculation, can serve for verification.

For both examples, the efficiency of the presented expressions and quality of the values obtained was also confirmed by comparing the obtained results with the results of the structural analysis program SAP2000, where the structures were discretized by a model with a large number of plane 2D finite elements. The computational models in program SAP2000 consisted of 2D four-nodded rectangular elements. In each element's node, two degrees of freedom were taken into account - vertical and horizontal displacements. The cracks were modelled by the exact discrete model, i.e. by non-connecting the nodes at the crack's surfaces. Concentrated loads as well as uniform loads were introduced as nodal forces. Vertical and horizontal displacements were obtained by implementing linear static analyses.

\subsection{First example - cracked cantilever beam with concentrated load at free end}

This example considers a cantilever of length $L=6 \mathrm{~m}$, Figure 2, with a rectangular cross-section of height of $0.50 \mathrm{~m}$ and width of $0.25 \mathrm{~m}$. For the material characteristics $E=30 \mathrm{GPa}$ and $v=0.3$ were selected.

The example was selected in order to demonstrate the utility of three-node finite element (CB3NFE) on a structure, which can also be discretized with a conventional two-node finite element (CB2NFE) whose third-degree polynomial interpolation functions were already proven to produce accurate values. The cantilever is loaded with a down-ward oriented concentrated force $F_{1}=10 \mathrm{kN}$. A crack of the depth of $0.25 \mathrm{~m}$ was introduced at a distance of $L_{1}=1 \mathrm{~m}$ from the support.

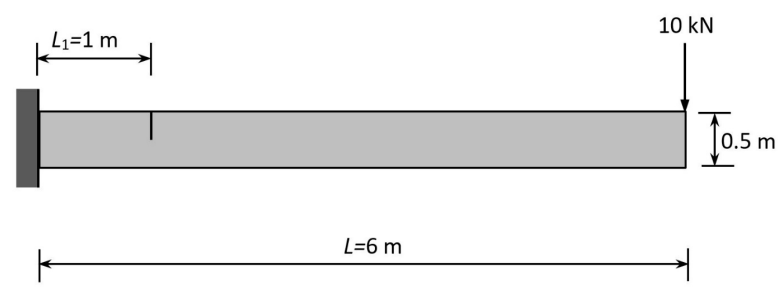

Figure 2: First example setup.

The initial discretization of the structure was done with a single CB3NFE finite element. Corresponding rotational spring $K_{r}=4.9093371 \cdot 10^{4} \mathrm{kNm}$ for the considered relative depth of the crack was calculated from Equation (1). The coefficients of the element's stiffness matrix were determined by utilization of Equation (20) and the computed stiffness matrix was: 
$\mathbf{K}_{1}=\left[\begin{array}{ccccc}9.10256 & 7.79294 & -9.5338 & 0.431241 & -0.846585 \\ 7.79294 & 6.87592 & -8.10063 & 0.307694 & -0.621455 \\ -9.5338 & -8.10063 & 10.0228 & -0.48902 & 1.01195 \\ 0.431241 & 0.307694 & -0.489023 & 0.0577814 & -0.16536 \\ -0.846585 & -0.621455 & 1.01195 & -0.16536 & 0.601671\end{array}\right] \cdot 10^{5}$

Since the complete structure was discretized by a single FE, the structure's stiffness matrix is identical to the finite element's stiffness matrix $\mathbf{K}_{1}$. Furthermore, by taking into account the boundary conditions in the left node, we can evaluate the discrete values of both unknown displacements and rotation in other nodes from the system of three linear equations:

$\left[\begin{array}{ccc}10.0228 & -0.48902 & 1.01195 \\ -0.489023 & 0.0577814 & -0.16536 \\ 1.01195 & -0.16536 & 0.601671\end{array}\right] \cdot 10^{5} \cdot\left\{\begin{array}{c}\mathrm{Y}_{2} \\ \mathrm{Y}_{3} \\ \Phi_{3}\end{array}\right\}=\mathbf{F}_{\mathrm{r}}$

where the reduced load vector of structure $\mathbf{F}_{\mathrm{r}}$ takes into the account the concentrated force on the right end:

$\mathbf{F}_{\mathrm{r}}=\left\{\begin{array}{c}0 \\ -10 \mathrm{kN} \\ 0\end{array}\right\}$

\section{Results and discussion}

The results for the discrete transverse displacements (obtained from the solutions of linear equations of the CB3NFE model) are together with the additional displacements in the middle of each elastic segment (obtained by the Equations (15) and (16)) summarized and demonstrated in the second column of Table 1. For further comparison, the third column shows the transverse displacements obtained by the two-node finite element of the cracked beam (CB2NFE) whereas the fourth column presents the displacements obtained by GDEs (Equation (28)). In addition to the simplified model's results, the transverse displacements obtained by an independent analysis of the structure in program SAP2000 are demonstrated in the fifth column. In this 2D FE model, 12,300 finite elements were implemented, and vertical and horizontal displacements were obtained in discrete points by solving 25,384 linear equations.

In the last column the results from the CB3NFE model with two elements (2FE) of equal lengths are presented. This additional discretization with CB3NFE model was prepared solely for two reasons. The first reason was to demonstrate that the derived at equations of CB3NFE element can be directly applied also to the non-cracked situations. Further, by implementing two finite elements it was thus demonstrated that a single CB3NFE element has already produced the exact values and, therefore, additional elements do not contribute to the accuracy of the computational model. This was additionally verified with several other discretizations, which are not presented since they have produced identical results.

The Table 1 demonstrates that the values of both beam models (CB3NFE and CB2NFE) in all presented points are identical and accurate compared to the values obtained from the GDEs. In comparison against the displacements obtained by 2D plane finite elements, the maximum $2.18 \%$ discrepancy was obtained at a distance of $3.5 \mathrm{~m}$ from the support, whereas the smallest discrepancy was obtained at the crack location and it was $0.14 \%$.

Table 1: Transverse displacements [mm] for some presented points.

\begin{tabular}{cccccc}
\hline \hline Location & CB3NFE & CB2NFE & GDEs & 12,300 2D FE & CB3NFE 2FE \\
\hline 0.5 m from left end & $-0.093333^{*}$ & $-0.093333^{* *}$ & -0.093333 & -0.094578 & $-0.093333^{*}$ \\
Crack & -0.362667 & $-0.362667^{* *}$ & -0.362667 & -0.36317 & -0.362667 \\
3.5 m from left end & $-6.335502^{*}$ & $-6.335502^{* *}$ & -6.335502 & -6.47696 & $-6.335502^{*}$ \\
Right end & -14.308337 & -14.308337 & -14.308337 & -14.5856 & -14.308337 \\
\hline
\end{tabular}

* obtained by utilization of the fourth-degree polynomial's interpolation functions of the CB3NFE model

** obtained by utilization of the third-degree polynomial's interpolation functions of the CB2NFE model 
In order to obtain the distribution of structure's transverse displacements, two functions must be analyzed for two elastic areas. These functions of the transverse displacements were determined by utilization of Equations (24) and (25):

$$
\begin{aligned}
& \mathrm{w}_{1}(\mathrm{x})=-3.84 \cdot 10^{-4} \mathrm{x}^{2}+2.1 \overline{33} \cdot 10^{-5} \mathrm{x}^{3} \quad(0 \leq \mathrm{x} \leq 1 \mathrm{~m}) \\
& \mathrm{w}_{2}(\mathrm{x})=1.018467 \cdot 10^{-3}-1.018467 \cdot 10^{-3} \mathrm{x}-3.84 \cdot 10^{-4} \mathrm{x}^{2}+2.1 \overline{33} \cdot 10^{-5} \mathrm{x}^{3} \quad(1 \mathrm{~m} \leq \mathrm{x} \leq 6 \mathrm{~m})
\end{aligned}
$$

The same transverse displacement functions were also obtained by utilization of the third-degree polynomial interpolation functions of the CB2NFE model. The transverse displacements can also be obtained by utilization of solution of GDEs (Equation (28)) for both elastic segments without transverse load $p$.

All three simplified model's transverse displacements functions (from CB3NFE and CB2NFE models as well as by governing differential equations solutions (GDEs)) are identical and accurate. This is generally valid for all examples without any continuous transverse load $p$.

In the next step additional analyses were conducted for various crack's locations and depths. Figure 3 a) thus demonstrates the transverse displacements of cantilever for various values of crack depth on location $1 \mathrm{~m}$ from left end. The results from four considered approaches (interpolated displacements of the CB3NFE model by utilization of the fourth degree polynomial functions, the CB2NFE model by utilization of polynomial functions of the third-degree, the solutions of GDE, likewise 12,300 2D plane finite elements model) are shown. Since all three solutions obtained by the simplified model are identical all three solutions for each relative crack depth are represented by a single line. The transverse displacements of non-cracked $\left(\delta^{\prime}=0\right)$ beam are also shown, exclusively to emphasize the effect of the introduced cracks. The largest transverse displacement's discrepancy (between simplified models and discrete values of the 12,300 2D FE model) was obtained for the situation with the relative crack depth ratio $\delta^{\prime}=0.5$, where the discrepancy at the free end of the cantilever was $1.9 \%$. Further, by reducing the relative crack depth ratio $\delta^{\prime}$, also the discrepancy of transverse displacements decreased simultaneously.

The influence of crack location on maximum transverse displacements was also analyzed for several crack locations (from $0.25 \mathrm{~m}$ to $5.75 \mathrm{~m}$ in $0.25 \mathrm{~m}$ step) for five values of relative crack depths (from $\delta^{\prime}=0.1$ to 0.5 ). The results are demonstrated in Figure $3 \mathrm{~b}$. The maximum discrete transverse displacement $Y_{3}=w_{\max }$ was obtained for the relative crack depth of $\delta^{\prime}=0.5$ on location $L_{1}=0.25 \mathrm{~m}$, which was $w_{\max }=-15.9506 \mathrm{~mm}$. Thus, the maximum obtained transverse displacement of the cracked cantilever in comparison with the non-cracked case increased for $73.08 \%$.

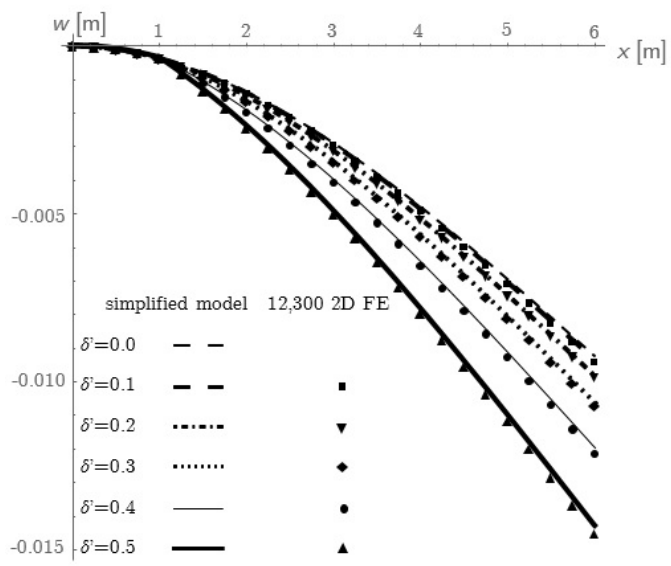

(a)

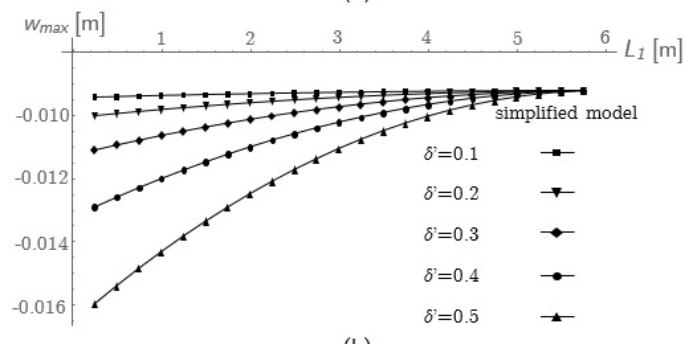

(b)

Figure 3: a) Influence of crack depth ratios on cantilever's transverse displacements for location of crack $L_{1}=1 \mathrm{~m}$. b) Influence of depth and location of crack on maximum displacement. 
Furthermore, the discrete bending moments and shear forces values in both end-nodes of the finite element were formally determined by utilization of the matrix algebra. However, due to the simplicity of the example, these values can be also straightforwardly evaluated by equilibrium equation.

The functions of the bending moments and shear forces were obtained from Equations (26) and (27). Since the transverse displacements functions for both beam models (CB3NFE and CB2NFE), as well as the solution of the differential equations are identical, it is clear that the functions of the bending moments and shear forces are also identical.

The discrete values obtained by summing nodal forces' moments from all plane elements, evaluated at the selected points along the cross section, give us excellent comparative values, since they were calculated completely independently of the obtained displacements. The values of the bending moments of the 2D plane model fully match the moments of the simplified model.

To complete the topic, the distribution of shear forces was also considered. Similar to the bending moments, the discrete values of the shear forces for 2D plane model were obtained by summing all the nodal vertical forces of the individual plane elements along the cross-section. These discrete values of shear forces also fully match the values of the simplified model.

\subsection{Second example - cracked propped cantilever with uniform load}

In the second example, a cracked propped cantilever was analyzed, Figure 4, loaded with a uniformly downward continuous load of $p=10 \mathrm{kN} / \mathrm{m}$ ' over the whole length of $L=10 \mathrm{~m}$.

This example was selected in order to demonstrate the utilization of the three-node finite element (CB3NFE) on a structure where the standard interpolation functions of the transverse displacements of the CB2NFE model (third degree polynomials) fail to produce exact solutions outside the element's nodes anymore.

The Young's modulus of the material was $E=30 \mathrm{GPa}$ with the Poisson coefficient $v=0.3$. The cross-section was a rectangle with height $h=0.4 \mathrm{~m}$ and width $b=0.25 \mathrm{~m}$. The crack with the relative depth of $\delta^{\prime}=0.5$ was located at a distance of $L_{1}=6 \mathrm{~m}$.

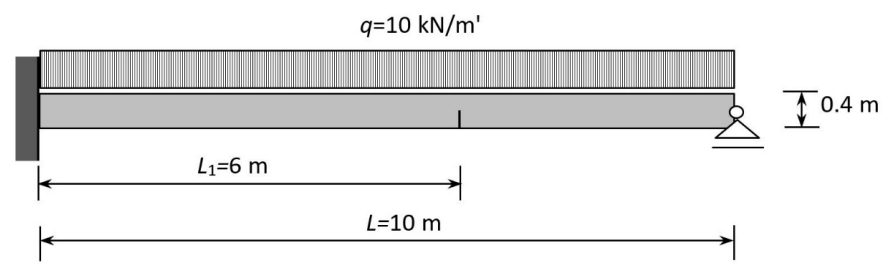

Figure 4: Second example setup.

The discretization of the structure was again executed with a single CB3NFE finite element, taking into account the value of the rotational stiffness $K_{r}=3.1419761 \cdot 10^{4} \mathrm{kNm}$ obtained from Equation (1). The stiffness matrix was determined by utilization of equation (20) and it is:

$\mathbf{K}_{1}=\left[\begin{array}{ccccc}0.13198 & 0.49639 & -0.25140 & 0.11942 & -0.18220 \\ 0.49639 & 2.35679 & -0.75535 & 0.25896 & -0.4143 \\ -0.25140 & -0.75535 & 0.74688 & -0.49548 & 1.22890 \\ 0.11942 & 0.25896 & -0.49548 & 0.37606 & -1.04670 \\ -0.18220 & -0.4143 & 1.22890 & -1.04670 & 3.50790\end{array}\right] \cdot 10^{4}$

whereas the corresponding load vector of the finite element due to the uniform load was obtained from Equation (23):

$\mathbf{F}_{1}=\left\{\begin{array}{c}-31.4679 \mathrm{kN} \\ -30.7587 \mathrm{kNm} \\ -53.6008 \mathrm{kN} \\ -14.9313 \mathrm{kN} \\ 1.6768 \mathrm{kNm}\end{array}\right\}$ 
For the chosen discretization, the finite element's stiffness matrix $\mathbf{K}_{1}$ also represents the stiffness matrix of the structure. Furthermore, by considering the boundary conditions in the left and right support, the discrete values $Y_{2}$ and $\Phi_{3}$ were evaluated from the system of two linear equations:

$\left[\begin{array}{ll}0.74688 & 1.22890 \\ 1.22890 & 3.50790\end{array}\right] \cdot 10^{4} \cdot\left\{\begin{array}{c}\mathrm{Y}_{2} \\ \Phi_{3}\end{array}\right\}=\mathbf{F}_{\mathrm{r}}$

where the reduced structure's load vector $\mathbf{F}_{\mathrm{r}}$ was obtained from the element's load vector $\mathbf{F}_{1}$ as:

$\mathbf{F}_{\mathrm{r}}=\left\{\begin{array}{c}-53.6008 \mathrm{kN} \\ 1.6768 \mathrm{kNm}\end{array}\right\}$

\section{Results and discussion}

Similar to the first example, the results of the transverse displacements obtained with three simplified models together with the 2D FE plane model were compiled (Table 2).

It can be perceived from Table 2 that the transverse displacements obtained by the CB3NFE model in the case of a uniform continuous load in all three selected locations are equal to the exact displacements obtained by the solution of the simplified model's governing differential equations (GDEs). On the other hand, substantial discrepancies are evident when comparing the CB2NFE model displacements' values against CB3NFE and GDEs' solutions. The maximum 54.3\% discrepancy occurs at a distance of $3 \mathrm{~m}$ from the clamped support and the smallest $27.0 \%$ in the middle of the second segment at a distance of $8 \mathrm{~m}$ from the clamped support. Furthermore, when the values of the CB3NFE model are compared with the 2D FE plane model's results, the discrepancy at the crack location is $1.53 \%$, whereas the discrepancy in the middle of the first segment is $1.82 \%$ and in the middle of the second segment is $1.76 \%$.

Table 2: Transverse displacements [mm] for some presented points.

\begin{tabular}{ccccc}
\hline \hline Location & CB3NFE & CB2NFE & GDEs & 16,440 2D FE \\
\hline 3 m from left end & $-8.895403^{*}$ & $-4.062654^{* *}$ & -8.895403 & -9.05988 \\
Crack & -17.12810 & $-9.951491^{* *}$ & -17.12810 & -17.435 \\
8 m from left end & $-11.04660^{*}$ & $-8.069442^{* *}$ & -11.04660 & -11.2188 \\
\hline \hline
\end{tabular}

* obtained by utilization of the fourth-degree polynomial's interpolation functions of the CB3NFE model

** obtained by utilization of the third-degree polynomial's interpolation functions of the CB2NFE model

In order to obtain the distribution of structure's transverse displacements along the structure, two functions for two elastic regions must be analyzed. The transverse displacements' functions for both elastic segments were determined from Equations (24) and (25), which took into the account the fourth-degree polynomial interpolation functions of the CB3NFE model:

$$
\begin{array}{rlr}
\mathrm{w}_{1}(\mathrm{x}) & =-1.6884757 \cdot 10^{-3} \mathrm{x}^{2}+2.646159 \cdot 10^{-4} \mathrm{x}^{3}-1.041 \overline{66} \cdot 10^{-5} \mathrm{x}^{4} & (0 \leq \mathrm{x} \leq 6 \mathrm{~m}) \\
\mathrm{w}_{2}(\mathrm{x}) & =-1.259757 \cdot 10^{-2}+2.099595 \cdot 10^{-3} \mathrm{x}-1.688476 \cdot 10^{-3} \mathrm{x}^{2} & \\
& +2.64616 \cdot 10^{-4} \mathrm{x}^{3}-1.041 \overline{66} \cdot 10^{-5} \mathrm{x}^{4} & (6 \mathrm{~m} \leq \mathrm{x} \leq 10 \mathrm{~m})
\end{array}
$$

Identical transverse displacement functions are obtained from Equation (28) by considering four boundary conditions and four continuity conditions, thus presenting the exact solutons of the simplified model's GDEs.

In the complementary 2D FE model vertical and horizontal displacements in discrete points were computed by solving 34,585 linear equations from the model consisting of 16,640 finite elements.

In Figure 5 the results of all four considered analyses are presented, where the results from CB3NFE interpolation functions and solutions of the GDEs appear as a single line. It can be further perceived that the transverse displacements of the CB3NFE model, likewise, the displacements obtained by the solutions of the GDEs almost perfectly fit the discrete values of the displacements of the 2D plane model at all points, whereas the transverse displacements function of the CB2NFE model evidently deviates from the exact values. Consequently, the functions of the bending moments and shear 
forces obtained from the second and third derivatives of the transverse displacements' functions of the CB2NFE model also deviate evidently from the exact values.

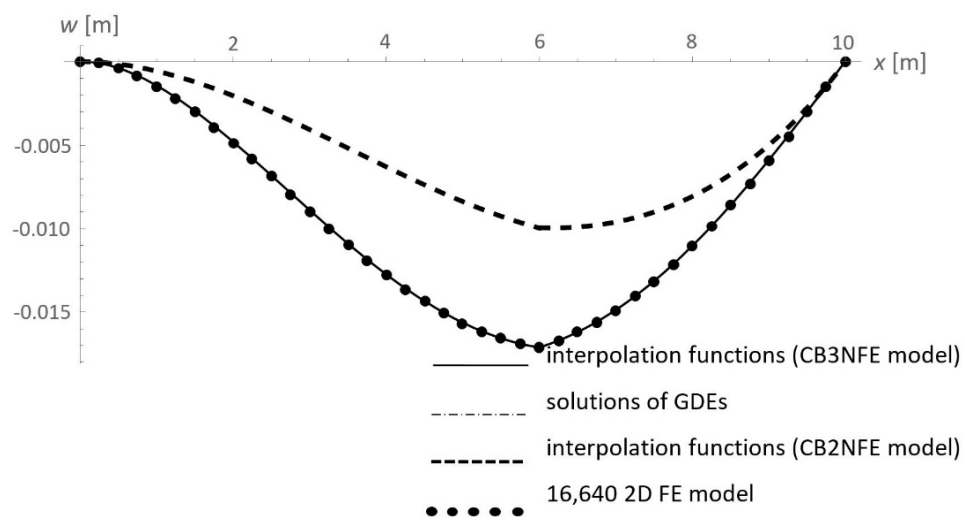

Figure 5: Transverse displacements along the x-axis.

Table 3: Internal forces-IF ( $M[\mathrm{kNm}]$ and $V[\mathrm{kN}])$ of both end-nodes.

\begin{tabular}{cccccc}
\hline \hline Location & IF & CB3NFE & CB2NFE & GDEs & 16,440 2D FE \\
\hline \multirow{2}{*}{ Left end $(\mathrm{x}=0)$} & $M$ & -135.0781 & -135.0781 & -135.0781 & -135.4845 \\
& $V$ & 63.50781 & 63.50781 & 63.50781 & 63.54837 \\
\hline \multirow{2}{*}{ Right end $(\mathrm{x}=10 \mathrm{~m})$} & $M$ & 0 & 0 & 0 & 0 \\
& $V$ & 36.49219 & 36.49219 & 36.49219 & 36.4516 \\
\hline \hline
\end{tabular}

The bending moments (M) and shear forces (V) in the two end nodes obtained from various approaches (CB3NFE and CB2NFE FE models, solutions of GDE, 2D plane FE model) are compared in Table 3.

From the comparison of the results of the nodal bending moments and shear forces in Table 3 obtained by the simplified model's solutions, it can be perceived that the values in the both ends are identical, and furthermore that these values exhibit just a moderate disagreement in comparison to the $2 \mathrm{D}$ model solutions.

The functions of bending moments and shear forces along the beam (Figure 6 and 7) were derived from the fourth degree interpolation functions, as well as from the second and the third derivatives of transverse displacements' functions (Equations (26) and (27)). Evidently, due to the correct functions of the transverse displacements in both approaches the functions of bending moments and shear forces are consequently perfectly matching.

The distribution of bending moments is demonstrated in Figure 6 . The values of the bending moments from the 2D plane model in the 11 equidistant discrete points along the beam are also added.

Similar to the first example, the values of shear forces of 2D plane model were obtained by summing all nodal forces tangential to the cross-section of each plane element. The bending moment's values were obtained by summing the contributions of the all moments caused by all nodal forces normal to the cross-section.

The values of the bending moments of the simplified CB3NFE model display a good agreement with the values of the $2 \mathrm{D}$ plane model at all selected discrete points. Thus, the discrepancy at the crack location is $0.25 \%$, at the middle of the first segment $2.8 \%$, which is the highest of all considered points, and at the middle of the second segment, the discrepancy is $0.15 \%$.

The shear forces of the simplified CB3NFE model also demonstrate excellent matching with the values of the 2D plane model at all measured points. The distribution of shear forces along the $x$-axis is demonstrated in Figure 7.

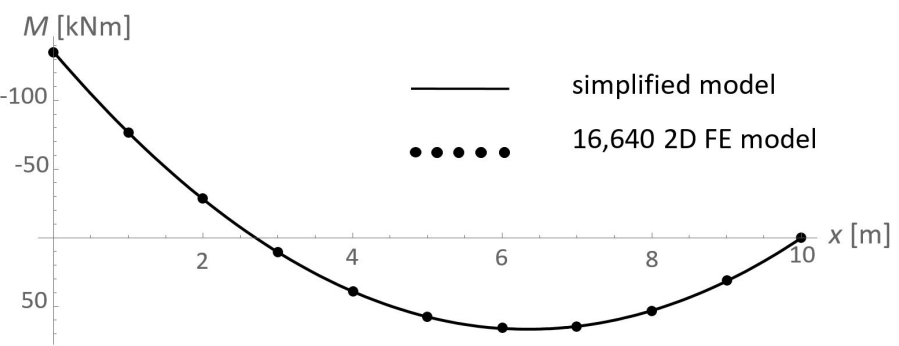

Figure 6: Distribution of bending moments along $x$-axis. 


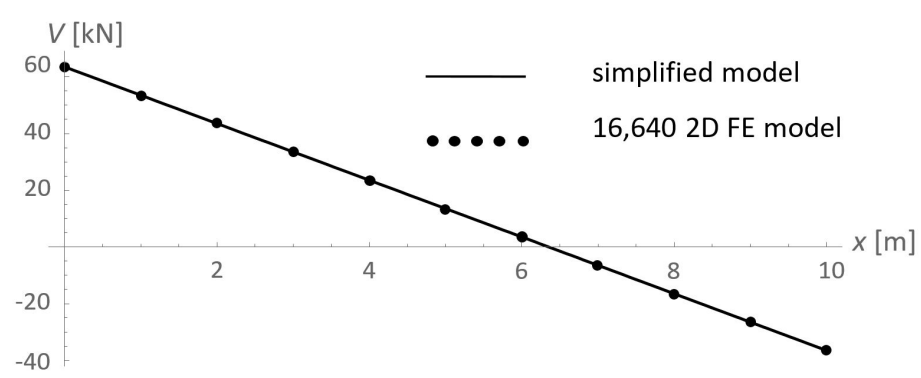

Figure 7: Distribution of shear forces along x-axis.

Of all the considered locations for shear forces, the maximum discrepancy at the location of the crack was $1.08 \%$, whereas in the middle of the first and second segment the discrepancies were only $0.11 \%$ and $0.23 \%$, respectively.

\section{CONCLUSIONS}

This article examines bending of cracked slender beam, where the transverse crack is represented by a linear rotational spring, which endows the hinge connecting two elastic parts. This simplified model was utilized to derive a new three-node finite element, which has, in addition to four standard degrees of freedom in both end nodes, an extra displacement at the location of the rotational spring as a degree of freedom. Thus, the third-degree polynomials, which were utilized as interpolation functions in previous approaches for the presentation of transverse displacements, were replaced by polynomials of the fourth degree.

The expressions for interpolation functions, the coefficients of the stiffness matrix, likewise the members of the load vector due to the uniform continuous load $p$ over the whole element, are obtained in closed symbolic forms. These derived terms can also be utilized in the case of a non-cracked beam.

Numerical examples demonstrate that the results obtained with the upgraded CB3NFE finite element fully match the results of the solution of the governing differential equation of the cracked beam in the case of a concentrated force, as well as in the case of a uniform continuous load over the entire length of the beam. Moreover, the presented finite element's results are in good agreement with the results obtained by the more detailed 2D plane finite elements' model as well.

The main novelty of the presented three nodded CB3NFE finite element in comparison to the previous two nodded model is that the transverse displacements' description (recently determined by the fourth-degree polynomial interpolation functions) is accurate in the case of a uniform continuous load over the entire finite element. The presented approach is proved to be ideal in modeling of cracked slender beams with uniformly continuous load. For example, it could be utilized in earthquake engineering according to the European standard EC8 for analysis of concrete elements where a cracked state should be considered.

Author's Contributions: Conceptualization, M Skrinar; Formal analysis, D Imamović; Investigation, D Imamović and M Skrinar; Methodology, D Imamović and M Skrinar; Project Administration, D Imamović; Software, D Imamović; Supervision, M Skrinar; Validation, D Imamović and M Skrinar; Writing - original draft, D Imamović; Writing - review \& editing, M Skrinar.

Editor: Marco L. Bittencourt.

\section{References}

Alijani A., Mastan M. A., Darvizeh A., Abadi M. Kh. (2018). Theoretical approaches for bending analysis of founded EulerBernoulli cracked beams. Archive of Applied Mechanics 88: 875 -895.

Biondi B., Caddemi S. (2007). Euler-Bernoulli beams with multiple singularities in the flexural stiffness. European Journal of Mechanics - A/Solids 26: 789-809.

Caddemi S, Caliò I. (2014). Exact reconstruction of multiple concentrated damages on beams. Acta Mechanica 225: 3137-3156.

Dimarogonas A. D., Papadopulus C. A. (1983). Vibration of cracked shafts in bending. Journal of Sound and Vibration 91: 583593. 
Gounaris G., Dimarogonas A. (1988). A finite element of a cracked prismatic beam for structural analysis. Computers \& Structures 28: 309-313.

Hasan W. M. (1995). Crack detection from the variation of the eigenfrequencies of a beam on elastic foundation. Engineering Fracture Mechanics 52: 409-421.

Mazanoglu K. (2015). A novel methodology using simplified approaches for identification of cracks in beams. Latin American Journal of Solids and Structures 12: 2460-2479.

Okamura H., Liu H. W., Chorng-Shin C., Liebowitz H. (1969). A cracked column under compression. Engineering Fracture Mechanics 1: 547-564.

Ostachowicz W. M., Krawczuk M. (1990). Vibration analysis of a cracked beam. Computers \& Structures 36: 245-250.

Palmeri A., Cicirello A. (2011). Physically-based Dirac's delta functions in the static analysis of multi-cracked Euler-Bernoulli and Timoshenko beams. International Journal of Solids and Structures 48: 2184-2195.

Rajab M. D., Al-Sabeeh A. (1991). Vibrational characteristics of cracked shafts. Journal of Sound and Vibration 147: $465-473$.

Skrinar M., Pliberšek T. (2004). New linear spring stiffness definition for displacement analysis of cracked beam elements. Proceedings in Applied Mathematics and Mechanics 4: 654-655.

Skrinar M. (2009). Elastic beam finite element with an arbitrary number of transverse cracks. Finite Elements in Analysis and Design 45: 181-189.

Skrinar M. (2013). Computational analysis of multi-stepped beams and beams with linearly-varying heights implementing closed-form finite element formulation for multi-cracked beam elements. International Journal of Solids and Structures 50 : 2527-2541.

Skrinar M., Pliberšek T. (2012). On the derivation of symbolic form of stiffness matrix and load vector of a beam with an arbitrary number of transverse cracks. Computational Materials Science 52: 253-260.

Skrinar M., Imamović D. (2018). Exact closed form finite element solution for the bending static analysis of transversely cracked slender elastic beams on Winkler foundation. International Journal for Numerical and Analytical Methods in Geomechanics 24: 1389 -1404.

Sundermeyer J. N., Weaver R. L. (1995). On crack identification and characterization in a beam by non-linear vibration analysis. Journal of Sound and Vibration 183: 857-871.

Tharp T. M. (1987). A finite element for edge-cracked beam columns. International Journal for Numerical Methods in Engineering 24: 1941-1950.

\section{APPENDIX A. Coefficients of interpolation functions.}

The coefficients $A_{i j}$, belonging to the interpolation functions' vector for the first elastic part (left of the crack), are given by utilization of Dirac $\delta$ functions as $(i, j=1,2, \ldots 5)$ :

$\mathrm{A}_{\mathrm{ij}}=\frac{1}{\chi} \sum_{\mathrm{k}=1}^{7} \sum_{\mathrm{l}=1}^{7} \mathrm{~L}^{1-1} \mathrm{~L}_{1}^{\mathrm{k}-1} \xi_{\mathrm{kl}}$

where the coefficients $\xi_{i j}$ are given as: 


$$
\begin{gathered}
\xi_{11}=\chi\left(\mathrm{d}_{11}+\mathrm{d}_{22}\right) ; \xi_{41}=4 \psi\left(\mathrm{d}_{15}-\mathrm{d}_{45}\right) ; \xi_{51}=2 \psi\left(-5 \mathrm{~d}_{14}+2 \mathrm{~d}_{25}+5 \mathrm{~d}_{44}-2 \mathrm{~d}_{55}\right) \\
\xi_{61}=2 \psi\left(3 \mathrm{~d}_{13}-5 \mathrm{~d}_{24}-3 \mathrm{~d}_{43}+5 \mathrm{~d}_{54}\right) ; \xi_{71}=6 \psi\left(\mathrm{d}_{23}-\mathrm{d}_{53}\right) ; \xi_{32}=12 \psi\left(-\mathrm{d}_{15}+\mathrm{d}_{35}\right) \\
\xi_{42}=-\frac{\xi_{41}}{2 \psi}+4 \psi\left(6 \mathrm{~d}_{14}-3 \mathrm{~d}_{25}-6 \mathrm{~d}_{34}+\mathrm{d}_{55}\right) ; \xi_{52}=2 \mathrm{~d}_{14}+24 \psi \mathrm{d}_{24}-2 \mathrm{~d}_{44}-10 \psi \mathrm{d}_{54} \\
\xi_{62}=6 \psi \mathrm{d}_{53} ; \xi_{33}=\frac{3 \xi_{41}}{4 \psi}+12 \psi\left(-\mathrm{d}_{14}+\mathrm{d}_{25}+\mathrm{d}_{34}\right) ; \xi_{43}=-\mathrm{d}_{25}-\mathrm{d}_{55}-12 \psi\left(-3 \xi_{17}+\mathrm{d}_{24}\right) \\
\xi_{53}=-\frac{3 \xi_{44}}{4}+\mathrm{d}_{24}+\mathrm{d}_{54} ; \xi_{14}=3 \xi_{32} ; \xi_{24}=-4 \psi\left(2 \mathrm{~d}_{14}+\mathrm{d}_{25}-2 \mathrm{~d}_{34}\right) \\
\xi_{34}=-4 \mathrm{~d}_{14}+2 \mathrm{~d}_{25}+4 \mathrm{~d}_{44}+\mathrm{d}_{55}+8 \psi\left(-6 \xi_{17}-\mathrm{d}_{24}\right) ; \xi_{44}=4 \mathrm{~d}_{13}+48 \psi \mathrm{d}_{23}-4 \mathrm{~d}_{43} \\
\xi_{54}=-2 \mathrm{~d}_{23}-\mathrm{d}_{53} ; \xi_{15}=\frac{\xi_{32}}{12 \psi}+3 \psi \xi_{16} ; \xi_{25}=-\mathrm{d}_{25}+6 \psi\left(3 \xi_{17}+\mathrm{d}_{24}\right) \\
\xi_{35}=-18 \psi \mathrm{d}_{23}-3 \mathrm{~d}_{24}-\mathrm{d}_{54} ; \xi_{45}=\mathrm{d}_{23}+\mathrm{d}_{53} ; \xi_{16}=2 \mathrm{~d}_{14}-\mathrm{d}_{34} \\
\xi_{26}=2 \mathrm{~d}_{24} ; \xi_{17}=-\mathrm{d}_{13}+\mathrm{d}_{33} ; \xi_{27}=-\mathrm{d}_{23} \\
\xi_{21}=\xi_{31}=\xi_{12}=\xi_{22}=\xi_{72}=\xi_{13}=\xi_{23}=\xi_{63}=\xi_{73}=\xi_{64}=\xi_{74}=\xi_{55}= \\
\xi_{65}=\xi_{75}=\xi_{36}=\xi_{46}=\xi_{56}=\xi_{66}=\xi_{76}=\xi_{37}=\xi_{47}=\xi_{57}=\xi_{67}=\xi_{77}=0
\end{gathered}
$$

with the following abbreviations:

$\chi=\mathrm{L} \cdot \mathrm{L}_{1}^{2}\left(\mathrm{~L}-\mathrm{L}_{1}\right)^{2}\left(\mathrm{~L}^{3}+12\left(\mathrm{~L}-\mathrm{L}_{1}\right) \mathrm{L}_{1} \psi\right)$

$\mathrm{d}_{\mathrm{ij}}=\delta_{\mathrm{ik}} \delta_{\mathrm{jl}}$

The remaining coefficients $B_{i j}(i, j=1,2, \ldots, 5)$ that belong to the interpolation functions for the second part can be further easily calculated by utilizing already determined coefficients $A_{i j}$ as:

$\mathrm{B}_{\mathrm{ij}}=\mathrm{A}_{\mathrm{ij}}+\left(-\delta_{1 \mathrm{j}}-\delta_{2 \mathrm{j}}\right)^{\mathrm{j}} \sum_{\mathrm{k}=3}^{5}\left(\prod_{\mathrm{l}=1}^{2}(\mathrm{k}-1)\right) \mathrm{L}_{1} \psi \mathrm{A}_{\mathrm{ik}}$ 